\title{
Responsabilidad social empresarial como estrategia de posicionamiento. Caso hoteles de la ciudad de Puyo
}

Corporate social responsibility as a positioning strategy. Hotels in the city

$$
\text { of Puyo }
$$

Chiriboga Ruilova Tania Marisol. ${ }^{1}$, Espín Ortíz Clímaco Geovani. ${ }^{2}$, Cevallos Punguil Tania Cristina. ${ }^{3} \&$ Jaramillo Cando Frumencio Stalin. ${ }^{4}$

\begin{abstract}
.
DOI: https://doi.org/10.33262/concienciadigital.v4i2.2.1781

This research addresses corporate social responsibility (CSR) as a positioning strategy in hotel establishments in the city of Puyo, including the development of new concepts that determine the ability of companies to approach and adopt CSR firsts as a corporate planning alliance and competitive advantage. Within the theoretical development, several parameters are established that served for the approach and performance of CSR in hotel establishments in basic public and private areas that determine conscious management with society, in addition to the importance that the public role has for CSR under policy or guidelines that promote this activation, also, the articulation of positioning strategies with instruments and mechanisms for their measurement was presented in the research. It is highlighted that the study was developed using an agile and critical methodology with a positivist approach of a comprehensive and constructivist nature through documentary analysis. Consequently, the strategies and processes proposed are essential

\footnotetext{
${ }^{1}$ Universidad Estatal Amazónica, Centro de Posgrado, Maestrante en Turismo, Mención Gestión del Turismo, Puyo-Ecuador, tanitakiss1994@hotmail.es, https://orcid.org/0000-0001-5855-6202

2 Universidad Estatal Amazónica, Facultad Ciencias de la Vida, Carrera de Turismo. Puyo, Ecuador. cespin@uea.edu.ec, https://orcid.org/0000-0002-7533-4924

3 Universidad Estatal Amazónica, Facultad Ciencias de la Vida, Carrera de Turismo, Puyo-Ecuador, tc.cevallosp@uea.edu.ec, https://orcid.org/0000-0002-7533-4924

${ }^{4}$ Universidad Técnica de Amato, Facultad de Ciencias de la Educación, Carrera de Cultura Física. Ambato, Ecuador. frumen112233@hotmail.com, https://orcid.org/0000-0003-4742-5172
} 
factors for an entity to be able to generate competition and positioning in a competitive market.

Keywords: Corporate social responsibility, Strategies, CSR Instruments, Positioning.

\section{Resumen.}

La presente investigación aborda la responsabilidad social empresarial (RSE) como estrategia de posicionamiento en establecimientos hoteleros de la ciudad de Puyo, incluido el desarrollo de nuevos conceptos que determinan la capacidad de las empresas para abordar y adoptar primicias de RSE como alianza de planeamiento corporativo y ventaja competitiva. Dentro del desarrollo teórico se establecen varios parámetros que sirvieron para el abordaje y actuación de la RSE en establecimiento hoteleros en áreas básicas públicas y privadas que determinan la gestión consciente con la sociedad, además de la importancia que el rol público posee para la RSE bajo política o lineamientos que promuevan esta activación, también, se presentó en la investigación la articulación de estrategias de posicionamiento con instrumentos y mecanismos para su medición. Se resalta que el estudio se desarrolló mediante una metodología ágil y critica con enfoque positivista de carácter comprensivo y constructivista a través de análisis documental. En consecuencia, las estrategias y procesos planteados se constituyen en factores indispensables para que una entidad pueda generar competencia y posicionamiento en un mercado competitivo.

Palabras claves: Responsabilidad social empresarial, Estrategias, Instrumentos de RSE, Posicionamiento.

\section{Introducción.}

\section{Responsabilidad Social Empresarial (RSE)}

$\mathrm{La}$ "responsabilidad social se define a menudo en un concepto en que las empresas integran elementos sociales y ambientales con las operaciones del negocio, gestión y relaciones con las partes interesadas" (Wulf Betancourt, 2018, pág. 14), de acuerdo con lo que afirma el autor la responsabilidad social consiste en un cumulo de acciones que deben ser adoptadas por las empresas para generar soluciones los problemas sociales que acogen los lugares en los cuales las empresas se desarrollan o tienen influencia directa e indirecta.

Las mociones de una empresa a lo largo del tiempo han cambiado y se conjugaron ya no solo en la creación de riqueza para sí mismas evolucionando de manera paulatina hasta la aparición de un concepto recurrente en la actualidad llamado Responsabilidad Social Corporativa lo que lleva a una empresa a asumir la responsabilidad del impacto que causan sus acciones en sus empleados, clientes, comunidad y medioambiente; la RSE integra en sus principios elementos sociales y ambientales en sus operaciones de negocio y gestión además de las relaciones que posee con las partes en las cuales interviene. 
Para Crane (2009) a quien citan (Sánchez, Cano, \& Peribáñez, 2017, pág. 23):“Se trata de comprender el impacto que genera la empresa en el entorno y considerar como puede utilizarse dicho impacto de forma positiva; Obviamente, positivo no solo para la empresa", mientras que para (Chávez, 2017) "La responsabilidad social corporativa no nos lleva a un estadio más benigno del capitalismo, nace para manejar riesgos en su reputación en un sistema de relaciones económica injusto” (pág. 88).

Es preciso mencionar que las Responsabilidad Social Corporativa al igual que la Responsabilidad Social Empresarial incurren en el mismo concepto, sin embargo, Wulf (2018) desarrolla una búsqueda más amplia en Google en la cual determina que en un periodo de 5 años 2011 al 2016 que el mayor número de búsquedas es a Corparate Responsibility con alrededor de 398 millones de entradas Business Social Responsibility con 144 millones de entradas, lo cual permite definir que los términos acuñados a la Responsabilidad Social Empresarial en búsqueda se fijan más en la responsabilidad corporativa con fines más extendidos que el aspecto social o dejando de lado al mismo.

\section{Áreas básicas de la RSE en el sector privado}

La Responsabilidad Social Empresarial comprende ciertas áreas o ámbitos que cubre para un desarrollo adecuado y bajo impacto en el accionar empresarial se destacan según (Rodríguez, 2019) derechos humanos, preocupaciones sociales, relaciones laborales y el medio ambiente las mismas que posee determinantes características conllevando mejoras para la empresa, sus colaboradores y la comunidad.

Cañas, (2018, pág. 14) distingue 5 áreas de RSE:

\section{Figura 1}

Áreas de gestión de RSE

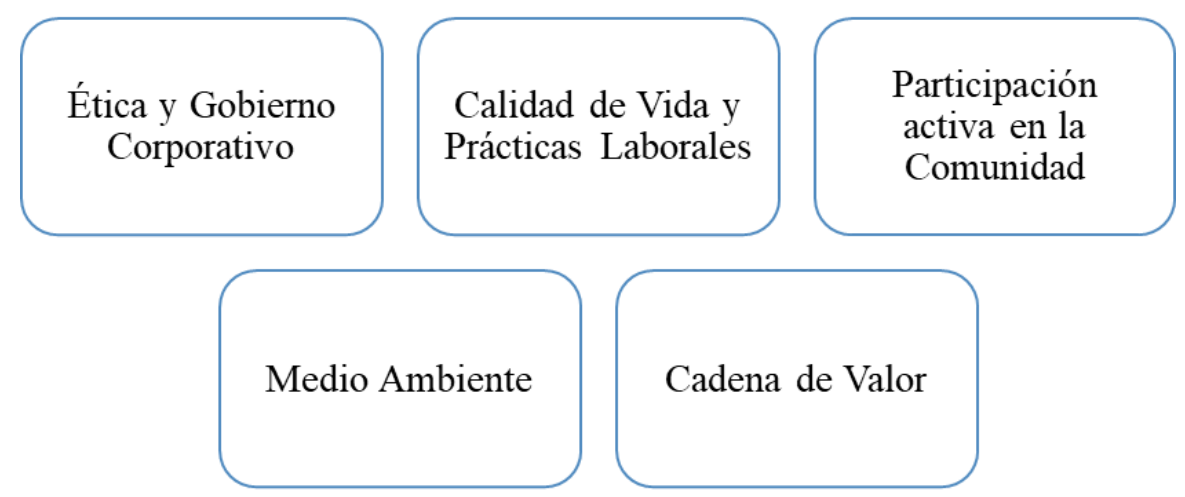

Nota. La figura representa las áreas de gestión de RSE en varios ámbitos. Tomado de Responsabilidad social corporativa, por Cañas, T., 2018, Elearning.

Con lo expuesto por Cañas es imprescindible terminar el valor que aporta cado una da las áreas en las cuales se encuentra inmersa la RSE, mediante la siguiente Tabla: 


\section{Tabla 1}

Descripción de las Áreas de gestión de RSE Área Descripción

Consiste en el conjunto de principios, procesos y objetivos estratégicos; en los cuales la empresa

Ética y Gobierno corporativo

Calidad de Vida y Práctica Laborales

Participación activa en la Comunidad

Medio Ambiente

Cadena de Valor basa sus creencias como la misión, visión y filosofía. Con el objetivo de reconocer todos los derechos civiles, políticos, económicos, sociales y culturales.

Incluye políticas de gestión de recursos humanos, compensaciones y beneficios sociales, promoción de carrera, gestión de talento, desarrollo personal, cuidado del ambiente y condiciones donde trabaja, promoción y cuidado de la salud, seguridad e higiene en el puesto de trabajo, promoción de la diversidad, garantía de la igualdad de género, promoción de estilos de vida saludable, etc.

Esta área genera iniciativas para maximizar el impacto social de sus contribuciones, para que se materialicen en dinero, tiempo, recursos, productos, servicios conocimientos, etc. Con la finalidad de mejorar la calidad de vida y desarrollo de la comunidad donde está implantada.

Principal compromiso de cada empresa socialmente responsable, lo cual implica cuidar el medio ambiente permanentemente y de manera consiente, así como los impactos en el ciclo de vida del producto y servicios minimizando posibles impactos negativos en la producción.

Según la ISO 26000 la cadena de valor "es la secuencia completa de actividades o partes que proporcionan valor (proveedores, trabajadores, contratistas, etc.) o reciben valor (clientes, consumidores, miembros y otros usuarios, etc.) en forma de productos o servicios". Incluyéndose áreas de marketing ventas entre otras que proporcionen valor.

Nota. Adaptado de Responsabilidad social corporativa (pág. 14), por Cañas, T., 2018, Elearning.

Con lo anteriormente expuesto se pone de manifiesto que el gran parte de la RSE se apoya en la gestión del talento humano y los subsistemas del mismo como se puede determinar a continuación:

\section{Tabla 2}

Subsistemas de talento humano y sus prácticas de RSE

\begin{tabular}{|c|c|c|}
\hline $\begin{array}{c}\text { Subsistemas de Talento } \\
\text { Humano (TH) }\end{array}$ & Contribución de TH a la RSE & $\begin{array}{c}\text { Contribución de la RSE a las } \\
\text { prácticasde TH }\end{array}$ \\
\hline Selección y reclutamiento & $\begin{array}{l}\text { - } \mathrm{TH} \text { puede chequear el } \\
\text { potencial de los empleados } \\
\text { con respecto a temas de RSE } \\
\text { TH puede seleccionar } \\
\text { personal que posea va- lores } \\
\text { alineados con los valores de } \\
\text { RSE de la empresa }\end{array}$ & $\begin{array}{l}\text { - RSE puede ayudar a atraer } \\
\text { mejorestalentos motivados } \\
\text { por los aspectos que } \\
\text { promueve en laempresa } \\
\text { - RSE promueve la selección } \\
\text { en THcon base en aspectos } \\
\text { como la diversidad y la }\end{array}$ \\
\hline
\end{tabular}


Evaluación y motivación

Compensación y sistema de recompensas

Capacitación y desarrollo
- TH puede promover compromiso con las prácticas y valores de RSE

- TH puede promover los valores de RSE para crear una cultura socialmente responsable en la empresa

- TH puede desarrollar esquemas de compensación a largo plazo.

- TH puede establecer incentivos que contribuyan a la consecución de los objetivos de RSE

- $\quad$ TH puede sensibilizar a los empleados en aspectos de RSE y ayudar a educarlos para que sean personas con mayor responsabilidad social
- RSE puede ayudar a evaluar las prácticas sociales de $\mathrm{TH}$ RSE puede ayudar a motivar al personal, promover el compromiso $\mathrm{y}$ la identificación con la empresa

- $\quad$ RSE puede ayudar a definir metas de TH que pueden ser usadas para incentivar y recompensar a los empleados.

- RSE puede ayudar a introducir estándares de trabajo digno.

- Gerentes de RSE pueden entrenar a los encargados de $\mathrm{TH}$ en el desarrollo de buenas prácticas.

Nota. Tomado de Franco, Espinoza, Pérez (2017)

Las áreas de RSE pueden variar de acuerdo a la estructura de las empres y por ende el tamaño lo que sugiere que disyuntivas que contribuyan a un mejor manejo de interno y externo de RSE, cada vez más comprometido con la sociedad y los impactos que pueda causar una empresa al mantener estándares de calidad que faculten criterios de obligatoriedad en la sociedad y su balance de impactos.

\subsection{Roles de la RSE en el sector público}

Los grandes compromisos de la RSE se han desarrollado en el mundo con el aporte de nuevas e innovadoras alternativas que pretenden un crecimiento socialmente responsable para las presentes y futuras generaciones con el seguimiento de varios organismos los cuales definen la RSE como un acto de compromiso corporativo para con los lugares a los cuales puede afectar su accionar, en el año 2003 el Banco Mundial menciona que se deben tener en cuenta políticas públicas de RSE con roles claros:

Tabla 3

Roles del sector público a nivel de RSE según el Banco Mundial

\begin{tabular}{ll}
\hline \multicolumn{1}{c}{ Roles } & \multicolumn{2}{c}{ La RSE del sector público para el Banco Mundial } \\
\hline Obligar & $\begin{array}{l}\text { Mando y control legal, Reguladores e Inspecciones, Sanciones y } \\
\text { recompensas. } \\
\text { Apoyo financiero, Sensibilización, Fomento de los mercados } \\
\text { Combinación de } \\
\text { recursos }\end{array}$ \\
Apoyo Político & Compromiso con partes Interesadas, Diálogo \\
\hline
\end{tabular}

Nota. Tomado de Banco Mundial (2014) a quien cita (Cueto, 2014, pág. 45) 
Las políticas que define el sector público en cuanto a la RSE recaen sobre los gobernantes del mundo, y por ende en el Ecuador mediante políticas de estado que garanticen las alternativas viables para la sociedad y la comunidad. De acuerdo con (Ormaza, Ochoa, Ramírez, y Quevedo, 2020, pág. 178) "la responsabilidad social empresarial en Ecuador a partir de la Agenda 2030, a través de una investigación con enfoque cualitativo utilizando la metodología de revisión documental, teórica y jurídica de las variables objeto de estudio".

Por tan motivo se expone criterios de RSE con base en lo tomado de las investigaciones anteriores en las cuales también los gobiernos son responsables del política y lineamientos claros que promuevan RSE adecuada y adaptada a principios de sostenibilidad social, policía, ambiental y cultural.

\subsection{Estrategias de posicionamiento empresarial mediante RSE}

Los principales mecanismos de Responsabilidad Social Empresarial se alimentan de las referencias internacionales, tal es el caso de la Organización Internacional del Trabajo (OIT) quienes apuntan con mecanismos que aportan a la RSE con métodos y procesos internos con relación en varios actores del territorio (Fernández, 2009).

Del mismo modo la Organización para la Cooperación y el Desarrollo Económicos (OCDE) plantea directrices enmarcada en estrategias de participación:

\section{Tabla 4}

Desarrollo de la estrategia de la RSE

\begin{tabular}{|c|c|c|c|c|}
\hline $\begin{array}{c}\text { Edades } \\
\text { económicas }\end{array}$ & $\begin{array}{c}\text { Característica de } \\
\text { la Rsc }\end{array}$ & $\begin{array}{c}\text { Modus } \\
\text { operandi }\end{array}$ & Factor clave & $\begin{array}{c}\text { Objetivo de las } \\
\text { partes interesadas }\end{array}$ \\
\hline Codicia & Defensiva & $\begin{array}{l}\text { Intervenciones } \\
\text { ad hoc }\end{array}$ & Inversiones & $\begin{array}{l}\text { Accionistas, } \\
\text { empleados, gobierno }\end{array}$ \\
\hline Filantropía & Caritativa & $\begin{array}{l}\text { Programas } \\
\text { caritativos }\end{array}$ & Proyectos & Comunidades \\
\hline Mercadeo & Promocional & $\begin{array}{l}\text { Relaciones } \\
\text { públicas }\end{array}$ & $\begin{array}{l}\text { Medios de } \\
\text { comunicación }\end{array}$ & Público en general \\
\hline Gestión & Estratégica & $\begin{array}{l}\text { Sistemas de } \\
\text { gestión }\end{array}$ & Códigos & $\begin{array}{l}\text { Accionistas y } \\
\text { ONG/Osc }\end{array}$ \\
\hline Responsabilidad & Sistémica & $\begin{array}{l}\text { Modelos } \\
\text { comerciales }\end{array}$ & Productos & $\begin{array}{l}\text { Reguladores y } \\
\text { clientes }\end{array}$ \\
\hline
\end{tabular}

Nota: Tomado de (Jung, 2018, pág. 11)

En gran medida la Responsabilidad Social Empresarial fija un horizonte en función de estratégicas la cuales de acuerdo con (Cortina, Arbeláez, y Villalba, 2017, pág. 55) quienes citan a (Filho, Wanderley, Gómez, Farache y Francisca, 2010; Husted, y Allen, 2000) mencionando que la RSE esta: "íntimamente relacionada con las estrategias empresariales y puede permitir a las empresas la generación de valor, mediante la creación o adquisición de ventajas competitivas y mediante la mejora de la reputación de la corporación".

Por ende, se resalta que las estrategias planteadas para RSE deben regirse a inmediatas y estrictas medidas para permitir sostenibilidad empresarial y comunitaria; a continuación, 
mediante la Figura se demuestra una ruta a seguir para lograr estrategias de dinamización en RSE:

\section{Figura 2}

Pasos para la generación de estrategias RSE

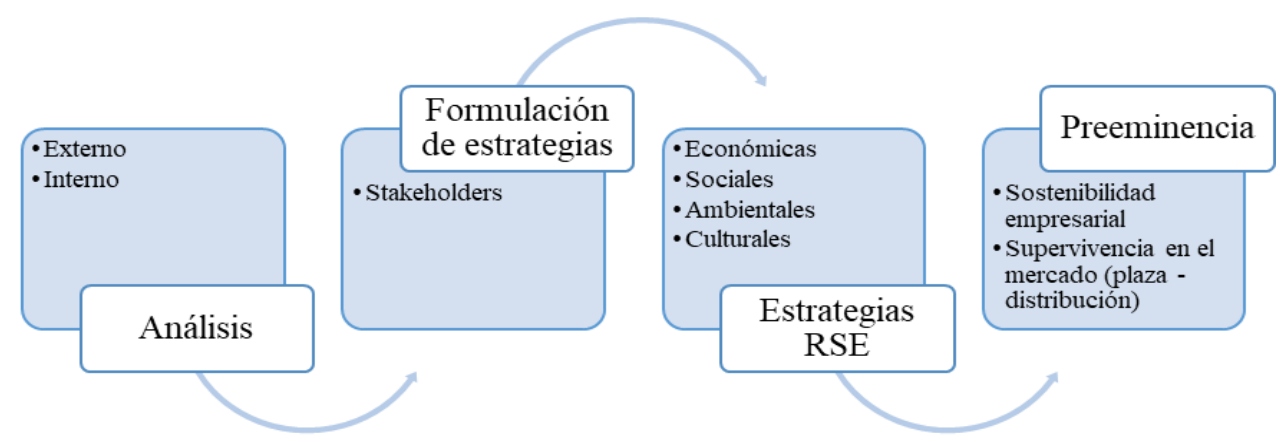

Una base para la generación de estrategias competitivas en función de la RSE es la detención de aspectos internos y externos de una empresa para fortalecer factores de riesgo y oportunidades de equilibro con lo cual se obtiene estabilidad corporativa con los actores del entorno, siendo importante determinar factores clave en la creación de vínculos colaterales y de participación para garantizar permanencia y posicionamiento.

\subsection{Instrumentos y mecanismos para la RSE}

La RSE contribuye a una medida para mitigar impactos negativos causados por las empresas quienes de manera voluntaria y autónoma asumen aplacar los efectos que pudiesen causar, apoyados en principio, estudios o lineamientos que les facultan para una gestión adecuada de RSE a través de mecanismo e instrumentos preelaborados o generados de manera autónoma por sí mismos.

Para Carroll (1991) a quien citan (Amezaga, Medina, Ramírez, \& Pineda, 2016, pág. 82)"sugiere que los cuatro tipos de responsabilidades sociales constituyen lo que en total podría llamarse RSE”, y presentan una pirámide con componente en la cual señala por orden de prioridad loas factores más consecuentes de la RSE:

\section{Figura 3}

Pirámide de la RSE

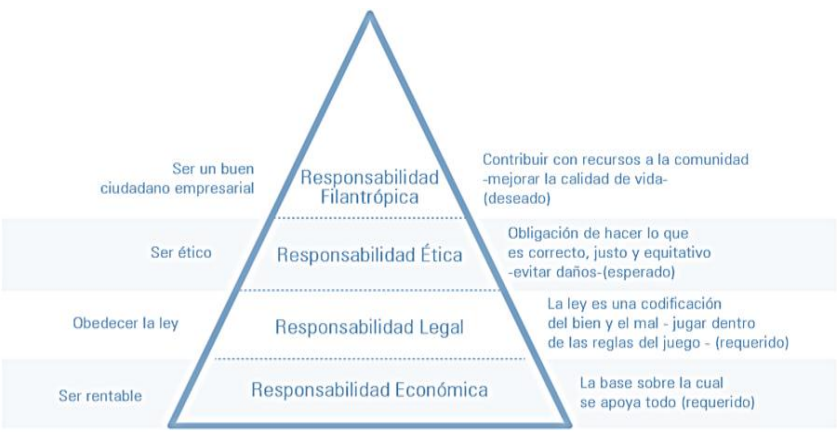

Nota. Tomado de Validación de un instrumento para medir la responsabilidad social empresarial en consumidores de México. Por Amezaga, Medina, Ramírez y Pineda, 2016, AD-minister, 70-100. 
Por otra parte, es indispensable medir los procesos y accionar del RSE de las organizaciones mediante dimensiones que permitan determinar su funcionalidad como lo mencionan los siguientes autores.

\section{Tabla 5}

Dimensiones reflejadas en las investigaciones $R S E$

\begin{tabular}{|c|c|c|c|}
\hline Autor & & Dimensiones RSE & $\begin{array}{c}\text { Relación positiva de laRSE } \\
\text { con: }\end{array}$ \\
\hline $\begin{array}{l}\text { Zapata } \\
\text { ySarache,2013 } \\
\text { (52) }\end{array}$ & $\begin{array}{l}\text { Clientes, } \\
\text { innovación }\end{array}$ & internos, & $\begin{array}{l}\text { Calidad (satisfacción al cliente, } \\
\text { procesos internos } \\
\text { innovación) }\end{array}$ \\
\hline
\end{tabular}

López, Ojeday

Ríos, 2017 (53)

Cubillos, Montealegro y Delgado, 2016

Urdaneta, 2014(45)

Pérez, Pérez y Añez, 2014 (46)

Martínez,

Monserrat

Serafín,

2015(44)

Wendlandtal., et 2016(47)

León, López y

Afcha,

Gómez, 2012

Sámano,

Salazar

yMendoza, 2014 (50)

Chimborazo, y otros, 2017 (51)
Derechos humanos, prácticas laborales, medio ambiente, prácticas justas de operación, asuntos de consumidores, participación activa, desarrollo de la comunidad, edad

Colaboradores, inversionista, clientes, proveedores, gobierno, comunidad y medio ambiente

Lineamientos estratégicos de RS, gobierno corporativo, capital humano, compras, clientes y consumidores, relaciones con el estado, desarrollo dela comunidad, gestión financiera, y gestión medioambiental

Ambiente, salud, nutrición, cultura, educación y deportes

Código de ética, prácticas laborales, medio ambiente y vinculación con los grupos de interés

Económica, legal, ética y filantrópica

Dirección corporativa, Empleados, Gobierno y sector público,Comunidad, Clientes, proveedores y competencia, Medio ambiente

Ética, RS

Ofrecer calidad, respetar al consumidor, participación activa con la comunidad, prácticas laborales y medio ambiente

Clientes, colaboradores, gobierno, ambiente medio

Desarrollo de lacomunidad y la gestión decompras

Deporte, la educación y salud

Calidad laboral, el medio ambiente mercadotecniasocial

Económica, legal, ética y

filantrópica

Bienestar de la comunidad, los servicios prestados al factor trabajo

Ética

Materia prima (reducción,reutilización, reciclaje, agroecológicos amigables con el medio ambiente); energía, agua, proveedores y cumplimiento normativo

Desarrollo sostenible: ambiental económica,social y

Alta intencionalidad decompra de los

RSE: económica, legal, ética consumidores
Materia prima yfilantrópica

Nota: Tomado de Bermudez y Mejías, (2018) 


\section{La RSE y los Establecimientos Hoteleros en el Puyo}

En el Ecuador es limitada la base Legal o legitimidad en la aplicación de la RSE en las empresas ecuatorianas, en la Constitución de la Republica del Ecuador los títulos más notables sobre responsabilidad social son: Título II: Derechos, Título VI: Régimen de desarrollo y el Título VII: Régimen del buen vivir. Además, la Ley Orgánica de Transparencia y Administración de la Información Pública, Ley de Gestión Ambiental, Ley de Protección al Consumidor, Código de Trabajo; en las cuales se habla de responsabilidad social y ambiental.

Además, la Universidad de las Americas, (2020) afirma lo siguiente sobre el aspecto legal de RSE en el país: "En el ámbito legal de Ecuador no existe una ley específica o un marco jurídico que obligue a todas las organizaciones a desarrollar proyectos de responsabilidad social empresarial", ya que se estaría violentando al principio de RSE de ser una práctica voluntaria.

Con estos antecedentes legales sobre RSE, dentro del estudio los establecimientos hoteleros integra una muestra que: “...construye con la visión económica tradicional, donde la empresa busca la rentabilidad maximizando sus beneficios económicos a través de factores como el incremento de la productividad y el mejoramiento de la calidad en el servicio hacia los clientes" (Sánchez, Vargas, Castillo, \& Zizumbo, 2018, pág. 47), con tal afirmación se ha considerado que la RSE sugestiona de sobre manera en la percepción del turista como consumidor. Para Sánchez, et. al., (2018) la RSE en empresas de alojamiento se sujeta a varias prácticas tales como: la calidad del servicio, gestión ambiental, coordinación gubernamental, condiciones laborales y vinculación con la comunidad; sin embargo en el Ecuador los enfoques de RSE pueden encajarse y adoptarse mediante un sistema de control el cual se ha actualizado para el registro de empresas turísticas que incluye a establecimientos hoteleros mediante Sistema Turístico Inteligente (SITURIN) vigente por acuerdo ministerial $\mathrm{N}^{\circ}$ 2021-005 plataforma de registro del Ministerio de Turismo en el cual se registra el certificado de responsabilidad social empresarial (MINTUR, 2021).

La RSE en empresas hoteleras parte de un concepto más dinámico puesto que son quienes objetivamente participan de un desarrollo económico que nace en el servicio lo cual infiere directamente en la sociedad como un sinónimo de responsabilidad cultural y ambiental, ya que base de su ingreso económico se debe a los atractivos turísticos y culturales que posee un lugar fuente motivadora de visitas turísticas aun lugar, en el Puyo la actividad turista está marcada por entornos naturales y culturales que gran demanda de turistas. Las investigaciones no arrojan resultados positivos sobre la existencia de RSE en este lugar por cual los registros carecen de notoriedad, un estudio en año 2018 el Gran Hotel Cofanes mediante enfoque sobre RSE (Castro y Chiriboga, 2018). 


\section{Figura 4}

RSE y principales stakeholders

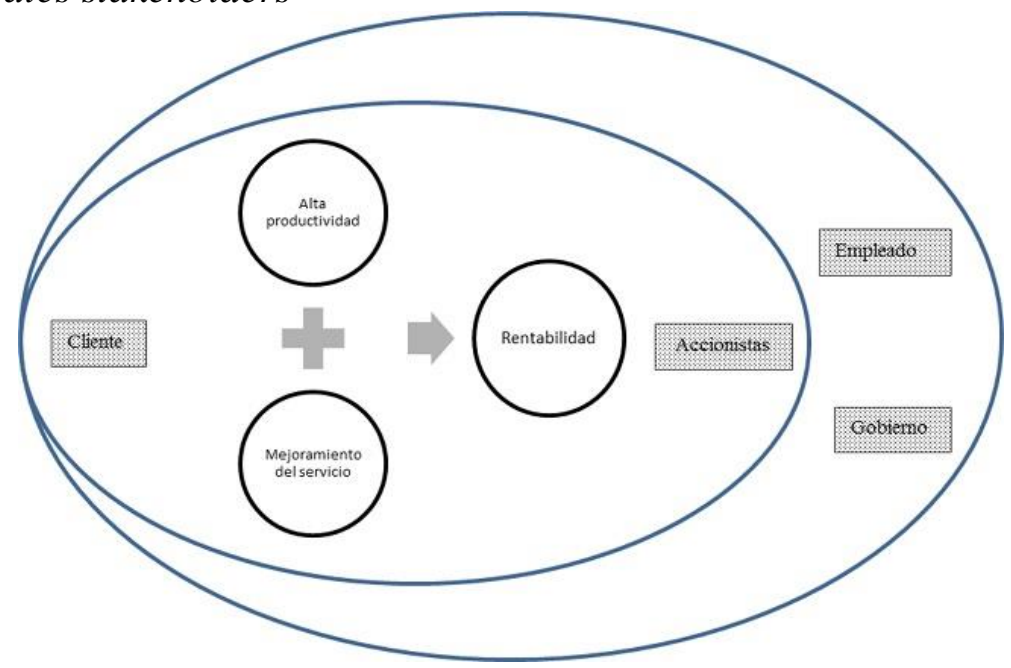

Nota. Tomado de Responsabilidad social corporativa. El papel de las Naciones Unidas y otras Organizaciones Internacionales en la Promoción de la RSC, Por Sánchez, Martínez, Nechar y Villarreal, 2018, DYKINSON.

A pesar de no contar con una certificación cuentan con medidas parciales de RSE mediante mejoramiento del servicio.

\section{Metodologia}

El estudio propuesto es de tipo exploratorio con un diseño transversal que permita explicar las condiciones que permanecen a las empresas en un periodo determinado. Con base en un estudio de caso en el área bajo el modelo business class que determina certificaciones ambientales y de gestión de la calidad de otras investigaciones para su validación como un modelo de gestión empresarial aplicable (Martínez, 2015).

A la investigación se integró bases de categorías de análisis a partir de resultados que forman parte de una investigación más amplia que integra la percepción de turistas y de empleados respecto a la responsabilidad social empresarial (RSE) en la ciudad del Puyo.

Por otra parte, en el desarrollo de la investigación se puntualiza el uso de un método documental para el avance adecuado del estudio se implementó desde una perspectiva teórica "que consiste en sustentar teóricamente el estudio, una vez que ya se ha planteado el problema de investigación” (Hernández, Fernández, y Baptista, 2014, pág. 61).

\section{Resultados}

La Responsabilidad Social Empresarial aporta nuevos conceptos en la industria hotelera, que en términos generales no se ha desarrollado en su totalidad en la localidad de estudio mediante investigación documentada se pudo resolver que únicamente está implícito en investigaciones como un aporte al sector hotelero local o propuesta de intención, lo cual sugiere la necesidad de un apoyo dinámico entre la empresa pública y privada para gestionar y mitigar impactos que pudiesen causar el desarrollo empresarial que fomente ganancias mediante estrategias aplicar. 
Surge la importancia empresarial y comprometida con la ciudad como agentes de solución a problemáticas presentes y futuras que determinen el rumbo de actuación a problemas globales capaces de reconfigurar el vínculo con la sociedad, no obstante están nuevos enfoques mediante cadenas de valor que innovan en el quehacer corporativo; desde un punto de vista empresarial la base de nuevos modelos y dimensiones RSE pueden permitirles lograr estrategias de dinamización económica con resultados positivos en otros ámbitos que protejan los recursos actuales y futuros.

De acuerdo con el catastro actualizado del año 2021 los 49 establecimientos hoteleros de la ciudad del Puyo no registran certificación de RSE dentro de los cuales se encuentran mediante categoría y clasificación: Casa de Huéspedes 2, Hostales de 1 Estrella 19, Hostales de 2 Estrellas 5, Hostales de 3 Estrellas 1, Hosterías de 3 Estrellas 11 y Hoteles de 2 Estrellas 11; de los cuales en ninguno refleja una certificación de registro de RSE. Sin embargo, se determinó en información documentada que el $10 \%$ de estos establecimientos se alinean en ciertos parámetros de RSE, tal es el caso del Gran Hotel Cofanes que posee un manual de RSE como aporte de investigaciones hechas en sus instalaciones por parte de investigadores del área turística.

\section{Tabla 6}

Clasificación de Establecimientos de alojamiento de acuerdo al análisis de sus prácticas de RSE en el Puyo

$$
\text { Clasificación de empresas hoteleras según sus prácticas de RSE }
$$

\begin{tabular}{|c|c|c|c|c|c|c|c|c|}
\hline \multirow{2}{*}{$\begin{array}{l}\text { Establecimientos } \\
\text { de Alojamiento }\end{array}$} & \multirow{2}{*}{$\begin{array}{c}\text { Tipo de } \\
\text { empresa }\end{array}$} & \multirow{2}{*}{ Tipo RSE } & \multirow{2}{*}{$\begin{array}{l}\text { Nivel } \\
\text { RSE }\end{array}$} & \multicolumn{4}{|c|}{ Valor cuantitativo } & \multirow{2}{*}{$\begin{array}{l}\text { Valor } \\
\text { cualitativo }\end{array}$} \\
\hline & & & & $\begin{array}{l}\text { Activ. } \\
\text { Econ. }\end{array}$ & $\begin{array}{l}\text { Activ. } \\
\text { Soc. }\end{array}$ & $\begin{array}{l}\text { Activ. } \\
\text { Amb. }\end{array}$ & Global & \\
\hline $\begin{array}{c}\text { Casa de } \\
\text { Huéspedes }\end{array}$ & $\begin{array}{c}\text { Reactiva } \\
\text { Filantrópica }\end{array}$ & Filantrópica & Mínimo & 2,1 & 1,8 & 1 & 1,63 & Deficiente \\
\hline $\begin{array}{c}\text { Hostales } 1 \\
\text { Estrella }\end{array}$ & $\begin{array}{c}\text { Reactiva } \\
\text { Filantrópica }\end{array}$ & Filantrópica & Mínimo & 2,2 & 1,9 & 1 & 1,73 & Deficiente \\
\hline $\begin{array}{c}\text { Hostales } 2 \\
\text { Estrellas }\end{array}$ & $\begin{array}{c}\text { Reactiva } \\
\text { Filantrópica }\end{array}$ & Filantrópica & Mínimo & 2,3 & 1,7 & 1,1 & 1,70 & Deficiente \\
\hline $\begin{array}{c}\text { Hostales } 3 \\
\text { Estrellas }\end{array}$ & $\begin{array}{l}\text { Reactiva } \\
\text { Filantrópica }\end{array}$ & Filantrópica & Mínimo & 2,5 & 2 & 1,1 & 2,33 & Deficiente \\
\hline $\begin{array}{c}\text { Hosterías } 3 \\
\text { Estrellas }\end{array}$ & $\begin{array}{c}\text { Reactiva } \\
\text { Filantrópica }\end{array}$ & Filantrópica & Bajo & 3,2 & 3 & 2,5 & 2,77 & Insuficiente \\
\hline $\begin{array}{l}\text { Hoteles } 2 \\
\text { Estrellas }\end{array}$ & Activa & Táctica & Medio & 2,5 & 2,5 & 2,1 & 2,93 & Aceptable \\
\hline
\end{tabular}

Nota. La clasificación de empresas hoteleras mediante el método clasificación permite medir el nivel de RSE con índices cualitativos y cuantitativos de acuerdo al tamaño de la entidad.

En la Tabla 6 mediante evaluación en función de su clasificación se determina la parcialidad de RSE en los establecimientos hoteleros del Puyo con un valor significativo del 2,93 considerado aceptable con base en investigaciones previas que afirman la postura del $10 \%$ de la totalidad de establecimientos hoteleros en el cantón que cumplen con RSE parcial sin identificación corporativa mediante registro o certificación.

\section{Discusión}

La referencia a lo cuestionado por la RSE incluida en las empresas u organizaciones, en la ciudad del Puyo permitiría una integración mediante metodologías ágiles capaces de 
integrar modelos en las áreas de negocio hotelero como estrategia de mercadeo para gestionar y liderar actuaciones congruentes con el fin de desarrollar estructuras organizativas y canalizar negocios sostenibles.

Los instrumentos y herramientas propuesta tienen el objetivo de estimular el comportamiento social responsable en las empresas y no se limitan únicamente a las de carácter turístico ya que los cuadros de mando permiten estandarización para medir el grado de RSC utilizando estrategias adaptables que permiten sintetizar el trabajo de las organizaciones.

En el sector de alojamiento el principal interés recurre en establecer vínculos o diálogos con cuatro de sus stakeholders o aliados estratégicos: los clientes, los accionistas, los empleados y el gobierno, un argumento que se ofrece en este sentido es que los accionistas mantengan roles indirectos y directos en el entorno empresarial ya que son ellos con quienes se canaliza el mando de la entidad junto con su fijación en la toman las decisiones administrativas y financieras.

Por otra parte, uno de los determinantes de discusión recae en el cliente, ya que en las empresas los factores como en reconocimiento público y ganar lealtad consisten elementos clave en el rol económico de la entidad como parte la RSE dando resultados prometedores en el caso de estudio el hallazgo del 10\% de RSE está bajo parámetros alineados a fidelizar y satisfacer al cliente estrechamente relacionados con los procesos de servicio considerando esto como agentes de valor agregado, confianza y fidelización.

Además, la RSE está ligada directamente con el buen desempeño de los empleados o el cliente interno quienes constituyen con la dinámica del proceso para logar el objetivo planteado que fija la estrategia a seguir mediante modelos RSE a aplicarse, junto con el cumplimiento legal de la empresa.

De tal modo que la verificación de criterios amplia la etapa de monitoreo con roles implícitos en metas fijadas por las empresas hoteleras de la localidad actualmente la principal preocupación se fija en las diversas alternativas de bioseguridad en medio de una pandemia dada por el Covid-19 con criterios que han cambiado la valoración de la RSE y demás estudio o análisis del turismo en el área de hospedaje que se van tomando en cuenta en la marcha con formatos de prueba y error, esto promueven resultados que coadyuvan a las entidades a sobrevivir fungiéndose más competitivas en el mercado, la investigación propone alternativas de aplicabilidad con metodologías agiles sujetas a indicaciones en medida de rangos palpables y diagnosticables.

\section{Conclusiones.}

- La Responsabilidad Social Corporativa y la Responsabilidad Social Empresarial recurren a una misma primicia que ratifica un poder social responsable que se adapta a los cambios sin deteriorar ningún recurso y/o producto, pero esto en teoría ya que en la práctica aún se ve difusa una solución de la interrogante 
manteniéndose los dos conceptos con parámetros distintos.

- En análisis de contexto cabe resaltar que la RSE mantiene estrecha relación con la comunidad creando un vínculo comercial responsable en el cual se tiene claro la primicia del cliente interno y externo con capacidad de generar una ventaja competitiva frente a otras empresas que no han desarrollado la RSE parcial.

- Los instrumentos y mecanismos de RSE permiten medir logros puntuales en el desarrollo de las prácticas de responsabilidad social y no todos recurren a la inversión de un capital económico, ya que ratifican medidas de gestión asociadas a social y capacitaciones del personal lo cual incurre en un ganar-ganar para las entidades.

- Las empresas hoteleras en la ciudad del Puyo requieren establecer RSE para generar mayor competencia y menos limitantes en su accionar como fuente de fidelización local empresarial, resaltando la diversidad de actividades sociales con las comunidades los cuales se convierten en portavoces de los servicios de las empresas logrando comunicación interna y consumo local.

\section{Referencias bibliográficas.}

Aldana, S., Vereda, F., Hidalgo-Alvarez, R., \& de Vicente, J. (2016). Facile synthesis of magnetic agarose microfibers by directed selfassembly. Polymer, 93, 61-64.

Amezaga, Medina, Ramírez, \& Pineda. (2016). Validación de un instrumento para medir la responsabilidad social empresarial en consumidores de México. AD-minister, 70-100.

Bermudez-Colina, Y., \& Mejías-Acosta, A. A. (2018). Medición de la responsabilidad social empresarial: casos en pequeñas empresas Latinoamericanas. Ingeniería Industrial, 315-325.

Bhat, S., Tripathi, A., \& Kumar, A. (2010). Supermacroprous chitosan-agarose-gelatin cryogels. in vitro characterization and in vivo assesment for cartilage tissue engineering. Journal of the Royal Society Interface, 1-15.

Bossis, G., Marins, J., Kuzhir, P., Volkova, O., \& Zubarev, A. (2015). Functionalized microfibers for field-responsive materials and biological applications. Journal of Intelligent Material Systems and Structures, 1-9.

Cañas, T. (2018). Responsabilidad social corporativa. Málaga: Elearning.

Castro, \& Chiriboga. (2018). Repositorio Universidad Estatal Amazónica. Recuperado el 18 de Febrero de 2021, de Repositorio Universidad Estatal Amazónica: https://repositorio.uea.edu.ec/xmlui/handle/123456789/451?locale-attribute=en 
Chávez, B. (2017). Tu consumo puede cambiar el mundo: El poder de tus elecciones responsables, concientes y críticas. Barcelona: Península.

Cortés, J., Puig, J., Morales , J., \& Mendizábal, E. (2011). Hidrogeles nanoestructurados termosensibles sintetizados mediante polimerización en microemulsión inversa. Revista Mexicana de Ingeniería Química., 10(3), 513-520.

Cortina, D. R., Arbeláez, C., \& Villalba, G. (2017). Responsabilidad social empresarial y construcción de la marca: una nueva mirada a las estrategias de gestión. Revista de Investigación, Desarrollo e Innovación, 49-60.

Cueto, C. (2014). ANÁLISIS DE LA RESPONSABILIDAD SOCIAL CORPORATIVA DE LAS GRANDES CIUDADES EN (Primera ed.). Santa Rosa: Área de Innovación y desarrolo S.L.

Dias, A., Hussain, A., Marcos, A., \& Roque, A. (2011). A biotechnological perspective on the application of iron oxide magnetic colloids modified with polysaccharides. Biotechnology Advances 29 , 29, 142-155.

Estrada Guerrero, R., Lemus Torres, D., Mendoza Anaya, D., \& Rodriguez Lugo, V. (2010). Hidrogeles poliméricos potencialmente aplicables en Agricultura. Revista Iberoamericana de Polímeros, 12(2), 76-87.

Fernández, R. (2009). Responsabilidad social corporativa. Una estrategia para conseguir imagen y reputación. Icono. Alicante: Club Universitario.

Franco, M. D., Espinoza Carrión, C. d., \& Pérez Espinoza, M. J. (2017). La responsabilidad social empresarial y su vínculo con la gestión del talento humano en las organizaciones. Revista Universidad y Sociedad, 9(1), 114-119. Recuperado el 19 de Febrero de 2021, de http://scielo.sld.cu/scielo.php?script=sci_arttext\&pid=S221836202017000100016\&lng=es\&tlng=es.

García-Cerda, L., Rodríguez-Fernández, O., Betancourt-Galindo, R., Saldívar-Guerrero, R., \& Torres-Torres, M. (2003). Síntesis y propiedades de ferrofluidos de magnetita. Superficies y Vacío., 16(1), 28-31.

Hernández Sampieri, R., Fernández Collado, C., \& Baptista Lucio, M. D. (2010). Metodologia de la investigacion (5ta Edicion ed.). México, México: McGRAWHILL / Interamericana Editores S.A. DE C.V.

Ilg, P. (2013). Stimuli-responsive hydrogels cross-linked by magnetic nanoparticles. Soft Matter, 9, 3465-3468.

Jung, S. (2018). Análisis de la rsc en un marco de desarrollo sostenible: la experiencia de la empresa coreana Samsung. Revista mexicana de estudios sobre la Cuenca del Pacífico, 12(24), 7-26. 
Lewitus, D., Branch, J., Smith, K., Callegari, G., Kohn, J., \& Neimark, A. (2011). Biohybrid carbon nanotube/agarose fibers for neural tissue engineering. Advanced Functional Materials, 21, 2624-2632.

Lin, Y.-S., Huang, K.-S., Yang, C.-H., Wang, C.-Y., Yang, Y.-S., Hsu, H.-C., . . Tsai, C.-W. (2012). Microfluidic synthesis of microfibers for magnetic-responsive controlled drug release and cell culture. PLOS ONE, 7(3), 1-8.

Martínez, E. E. V. (2015). Responsabilidad social empresarial y gestión ambiental en el sector hotelero. Universidad Autónoma del Estado de México.

Ministerio de Turismo del Ecuador MINTUR. (10 de Febrero de 2021). Ministerio de Turismo del Ecuador. Recuperado el 28 de Abril de 2021, de Ministerio de Turismo del Ecuador MINTUR Web Site: https://siturin.turismo.gob.ec/login

Ormaza Andrade, J., Ochoa Crespo, J., Ramírez Valarezo, F., \& Quevedo Vázquez, J. (2020). Responsabilidad social empresarial en el Ecuador: Abordaje desde la Agenda 2030. Revista de Ciencias Sociales, XXVI(3), 175-193.

Rodríguez, A. (2019). Responsabilidad social corporativa. ADGG072PO (Primera ed.). Málaga: ic.

Ruiz Estrada, G. (2004). Desarrollo de un Sistema de liberación de fármacos basado en nanopartículas magnéticas recubiertas con Polietilénglicol para el tratamiento de diferentes enfermedades. Madrid: Universidad Autónoma de Madrid. Departamento de Física Aplicada.

Sánchez, H., Vargas, E., Castillo, M., \& Zizumbo, L. (2018). Responsabilidad Social Empresarial en la Hotelería. Un Enfoque Ético. Gestão e Regionalidade, 34(102), 43-57. doi:10.13037/gr.vol34n102.4356

Sánchez, V., Cano, M. Á., \& Peribáñez, E. (2017). Responsabilidad social corporativa. El papel de las Naciones Unidas y otras Organizaciones Internacionales en la Promoción de la RSC. Madrid: DYKINSON.

Universidad de las Américas. (22 de Abril de 2020). Universidad de Las Américas | Diseño y Desarrollo Marketing Digital. Recuperado el 21 de Diciembre de 2020, de Universidad de Las Américas | Diseño y Desarrollo Marketing Digital Web Site: https://marketing.udla.edu.ec/comunicacion/responsabilidad-social-enecuador/

Wulf Betancourt, E. (2018). Responsabilidad Social Empresarial: Un desafío corporativo (Primera ed.). La Serena: Universidad de la Serena.

Wulff-Pérez , M., Martín-Rodriguez, A., Gálvez-Ruiz, M., \& de Vicente, J. ( 2013 ). The effect of polymer surfactant on the rheological properties of nanoemulsions. Colloid and Polymer Science, 291, 709-716. 
Zamora Mora, V., Soares, P., Echeverria, C., Hernández, R., \& Mijangos, C. (2015). Composite chitosan/Agarose ferrogels for potential applications in magnetic hyperethermia. Gels., 1, 69-80.

1 Ciencia 


\section{PARA CITAR EL ARTÍCULO INDEXADO.}

Tania Marisol, C. R., Clímaco Geovani, E. O., Tania Cristina, C. P., \& Frumencio Stalin, J. C. (2021). Responsabilidad social empresarial como estrategia de posicionamiento. Caso hoteles de la ciudad de Puyo . ConcienciaDigital, 4(2.2), 103-119. https://doi.org/10.33262/concienciadigital.v4i2.2.1781

\section{Ciencia \\ LDigital}

El artículo que se publica es de exclusiva responsabilidad de los autores y no necesariamente reflejan el pensamiento de la Revista Conciencia Digital.

El artículo queda en propiedad de la revista y, por tanto, su publicación parcial y/o total en otro medio tiene que ser autorizado por el director de la Revista Conciencia Digital.

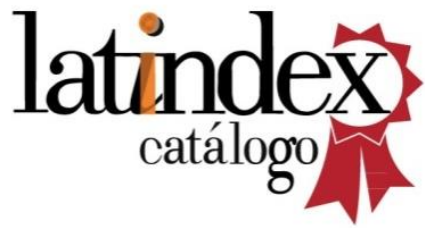

